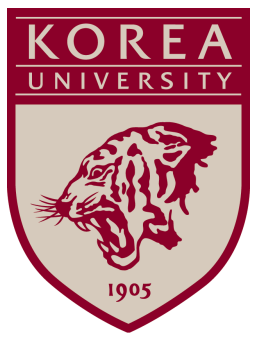

Discussion Paper Series

No. 1506

July 2015

\title{
An Improvement to Jensen's Inequality and its Application to Mating Market Clearing when Paternity is Uncertain
}

\author{
Dirk Bethmann
}

The Institute of Economic Research - Korea University

Anam-dong, Sungbuk-ku, Seoul, 136-701, South Korea, Tel: (82-2) 3290-1632, Fax: (82-2) 928-4948

Copyright (C) 2015 IER. 


\title{
An Improvement to Jensen's Inequality and its Application to Mating Market Clearing when Paternity is Uncertain*
}

\author{
Dirk Bethmann ${ }^{\dagger}$
}

July 31,2015

\begin{abstract}
Jensen's inequality can be tightened in the context of binomially distributed random variables. The suggested new inequality coincides with Jensen's inequality only when the expected value of the random variable is an integer, in all other cases the inequality is tighter than Jensen's. This improvement is used to derive a welfare result in the context of a mating market where male agents are exposed to paternal uncertainty. It is shown that social welfare is highest when paternity is certain, a result that can not be obtained when relying on Jensen's inequality.
\end{abstract}

JEL Classification: C65; J11; J13.

Keywords: Expected Utility, Binomial Distribution, Jensen's Inequality, Mating Market, Uncertain Paternity.

\section{Introduction}

In many applications, economic variables are discrete by their very nature. However, because discrete variables can cause theoretical difficulties economists often decide to use their continuous counterparts. This is true even for the most basic demand and supply functions as they are studied in undergraduate classes where the assumption of continuity might be completely innocuous. In some contexts, however, it might be advisable to keep the discrete nature of a variable. In this case, deriving results that hold under some general assumptions becomes much more difficult, particularly when the variable is also random.

In this note, I will derive an upper bound for expected utility when the variable of interest is drawn from a binomial distribution. Consider, for example, the number of children

\footnotetext{
${ }^{*}$ This research is supported by a Korea University Grant (K1420651). All remaining errors are my own.

${ }^{\dagger}$ Korea University, Anam-dong, Sungbuk-gu, Seoul 136-701, Republic of Korea email: dirk@korea.ac.kr
} 
in a fertility model with uncertain paternity (Bethmann and Kvasnicka, 2011). There a man's number of own offspring $(k)$ follows a binomial distribution with parameters $n$ and $\delta .{ }^{1}$ Assuming that a man's utility from own offspring is described by a strictly concave function $v(x)$, one might be interested in an upper bound for his expected utility. One could, of course, simply apply Jensen's inequality:

$$
\mathbb{E}[v(k)] \leq v(\mathbb{E}[k]) \quad \text { with } \quad \mathbb{E}[v(k)]<v(\mathbb{E}[k]) \quad \text { if } \quad \mathbb{P}[k \neq \mathbb{E}[k]]>0
$$

For example, with $n=3$ and $\delta=0.6$ the expected number of own offspring is $\mathbb{E}[k]=1.8$ and hence $\mathbb{E}[v(k)]<v(1.8)$. However, we will show that this upper bound for expected utility can be further lowered. The following calculations illustrate the intuition. Expected utility $\mathbb{E}[v(k)]$ is given by:

$$
\mathbb{E}[v(k)]=\frac{8}{125} v(0)+\frac{36}{125} v(1)+\frac{54}{125} v(2)+\frac{27}{125} v(3)
$$

Because $v(0)+v(3)<v(1)+v(2)$ holds for any strictly concave function $v$, one can eliminate the first term on the right hand side:

$$
\mathbb{E}[v(k)]<\frac{8-8}{125} v(0)+\frac{36+8}{125} v(1)+\frac{54+8}{125} v(2)+\frac{27-8}{125} v(3)=\frac{44}{125} v(1)+\frac{62}{125} v(2)+\frac{19}{125} v(3)
$$

Similarly, one can eliminate the last term (because $v(1)+v(3)<2 v(2)$ holds):

$$
\mathbb{E}[v(k)]<\frac{44-19}{125} v(1)+\frac{62+38}{125} v(2)+\frac{19-19}{125} v(3)=\frac{1}{5} v(1)+\frac{4}{5} v(2)
$$

Clearly, $0.2 v(1)+0.8 v(2)<v(1.8)$ holds, such that the upper limit in (4) is indeed lower than the value implied by Jensen's inequality. Two points in this instructive example are worth emphasizing. First, at each step we have reduced the number of relevant values of $k$ in the process of determining the upper limit by one and we stopped when there were only two values $(k=1$ and $k=2)$ left. As will be shown in Section 2, this carries over to the general case. ${ }^{2}$ Second, the two remaining values are neighboring the expected value. To be precise, they are the largest integer not exceeding $\mathbb{E}[k]$ and the smallest integer not less than $\mathbb{E}[k]$. Again, this feature carries over to the general case.

Figure 1 plots the strictly concave function $v(x)$. Function values $f(k)$ with $k \in\{0,1,2,3\}$ as used in (2) are highlighted by the black points. Linearity of the expectation operator allows us to neglect all function values $f(x)$ with $x \in \mathbb{R} \backslash\{0,1,2,3\}$ and to focus instead on the piecewise linear curve shown in grey. However, most line segments are not of interest. As was shown in (3) and (4), one can use concavity and the weights from the binomial distribution to eliminate most line segments except for the segment that contains the expected value $\mathbb{E}[k]$. In the proof of our main result, we will later introduce function $s(x)$ which extrapolates this remaining segment and then serves as an upper bound for $f(k)$ (dashed line in Figure 1).

\footnotetext{
${ }^{1}$ Parameter $n$ is the number of females the man has mated and $\delta$ is the probability of fatherhood.

${ }^{2}$ In some cases it is possible to reduce even further to just one relevant value for $k$. However, as it turns out, the suggested inequality coincides with Jensen's in those cases, i.e. the only remaining value is $\mathbb{E}[k]$.
} 
Figure 1: Strictly concave utility function $\mathrm{v}(\mathrm{x})$ and its secant $\mathrm{s}(\mathrm{x})$.

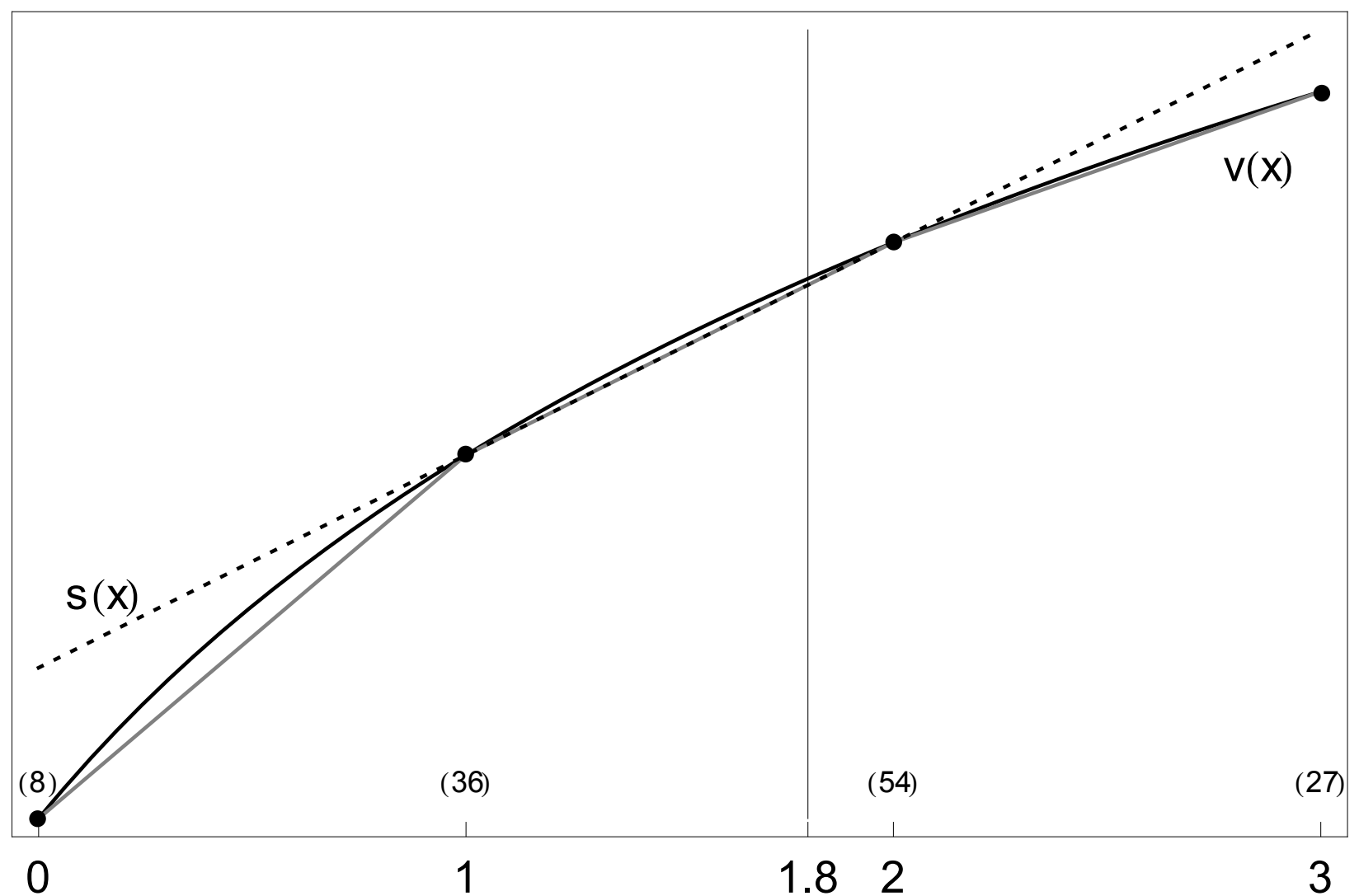

Notes: The black points denote the utility levels $v(k)$ for each $k \in\{0,1,2,3\}$. The numbers in parentheses represent the weights of each value of $v(k)$ in $\mathbb{E}[v(k)]$ as used in equation (2).

\section{Using the Interval of Weak Medians to improve Jensen's Inequality}

Before we present the main result, we need to introduce some notation. Let $x \in \mathbb{R}$ be given. The floor function $\lfloor x\rfloor$ gives the largest integer not exceeding $x$. Similarly, the ceiling function $\lceil x\rceil$ yields the smallest integer not less than $x$ :

$$
\lfloor x\rfloor=\max \{m \in \mathbb{Z} \mid m \leq x\} \quad \text { and } \quad\lceil x\rceil=\min \{n \in \mathbb{Z} \mid n \geq x\}
$$

The sawtooth function $\{x\}$ gives the fractional part of $x$ and can be defined in terms of the floor function: ${ }^{3}$

$$
\{x\}=x-\lfloor x\rfloor
$$

\footnotetext{
${ }^{3}$ Unfortunately, conventional notation uses braces both to denote the sawtooth function and to identify the elements of a set. The reader must glean from the context what is meant.
} 
For example, consider the case where $x=1.8$, then $\lfloor 1.8\rfloor=1,\lceil 1.8\rceil=2$ and $\{1.8\}=0.8$. We are now equipped to present the main result.

TheOREM. Let function $f(x)$ with $x \in \mathbb{R}$ be concave. Consider the expected value $\mathbb{E}[f(k)]$ of function $f(k)$ under the binomial distribution with $n \in \mathbb{N}$ draws and probability of success $\delta \in(0,1)$ with $k \in\{0, . ., n\}$. Then the following inequality holds:

$$
\mathbb{E}[f(k)] \leq\{n \delta\} f(\lceil n \delta\rceil)+(1-\{n \delta\}) f(\lfloor n \delta\rfloor)
$$

For strictly concave function $f$, the inequality in (7) is strict when $n>1$.

Proof. When $n \delta$ is an integer (i.e. $n \delta \in \mathbb{N}$ ), the weak inequality in (7) reduces to Jensen's inequality, i.e. $\mathbb{E}[f(k)] \leq f(\mathbb{E}[k])=f(n \delta)$. Hence, let us assume that $n \delta \notin \mathbb{N}$ holds. In that case:

$$
0<\{n \delta\}<1 \quad \text { and } \quad\lceil n \delta\rceil-\lfloor n \delta\rfloor=1
$$

follow from the properties of the floor, ceiling and sawtooth functions. Consider the linear function $s(x)$ through points $(\lfloor n \delta\rfloor, f(\lfloor n \delta\rfloor))$ and $(\lceil n \delta\rceil, f(\lceil n \delta\rceil))$ :

$$
s(x)=m x+b, \quad \forall x \in \mathbb{R}
$$

where the slope $m$ and the intercept $b$ are given by:

$$
m=f(\lceil n \delta\rceil)-f(\lfloor n \delta\rfloor) \quad \text { and } \quad b=f(\lfloor n \delta\rfloor)\lceil n \delta\rceil-f(\lceil n \delta\rceil)\lfloor n \delta\rfloor
$$

Note that $s(x)$ is a secant with the property that no point of $f$ is above $s$ for any $k \in\{0, . ., n\}$ :

$$
s(k) \geq f(k), \quad \forall k \in\{0, \ldots, n\}
$$

To show this, consider the case where $0 \leq k \leq\lfloor n \delta\rfloor$ holds. The proof is by contradiction, i.e. assume that $s(k)<f(k)$ holds, then:

$$
f(\lceil n \delta\rceil)(k-\lfloor n \delta\rfloor)+f(\lfloor n \delta\rfloor)(\lceil n \delta\rceil-k)<f(k)
$$

from the construction of the secant $s(x)$. Rearranging yields:

$$
f(\lfloor n \delta\rfloor)<\frac{1}{\lceil n \delta\rceil-k} f(k)+\frac{\lfloor n \delta\rfloor-k}{\lceil n \delta\rceil-k} f(\lceil n \delta\rceil)
$$

The contradiction follows from $\frac{1}{\lceil n \delta\rceil-k} k+\frac{\lfloor n \delta\rfloor-k}{\lceil n \delta\rceil-k}\lceil n \delta\rceil=\lfloor n \delta\rfloor$ and the concavity of function $f$. For the case $\lceil n \delta\rceil \leq k \leq n$, we also assume that $s(k)<f(k)$ holds, so:

$$
f(\lceil n \delta\rceil)(k-\lfloor n \delta\rfloor)+f(\lfloor n \delta\rfloor)(\lceil n \delta\rceil-k)<f(k)
$$

Rearranging, we obtain:

$$
f(\lceil n \delta\rceil)<\frac{1}{k-\lfloor n \delta\rfloor} f(k)+\frac{k-\lceil n \delta\rceil}{k-\lfloor n \delta\rfloor} f(\lfloor n \delta\rfloor)
$$


This time $\frac{1}{k-\lfloor n \delta\rfloor} k+\frac{k-\lceil n \delta\rceil}{k-\lfloor n \delta\rfloor}\lfloor n \delta\rfloor=\lceil n \delta\rceil$ holds, which is a contradiction for concave function $f$. Hence, we have shown that $s(k) \geq f(k)$ holds for all $k \in\{0, . ., n\}$. Given the properties of the expectations operator, it holds that:

$$
\mathbb{E}[f(k)] \leq \mathbb{E}[s(k)]=m \mathbb{E}[k]+b=m n \delta+b
$$

From $n \delta=\lfloor n \delta\rfloor+\{n \delta\}(\lceil n \delta\rceil-\lfloor n \delta\rfloor)$, and the definitions of $m$ and $b$ in equation (10), we obtain the desired result:

$$
\mathbb{E}[f(k)] \leq\{n \delta\} f(\lceil n \delta\rceil)+(1-\{n \delta\}) f(\lfloor n \delta\rfloor)
$$

To show the strictness in (7) for strictly concave $f(x)$ when $n>1$, it suffices to show that there is at least one $k \in\{0, . ., n\}$ where (11) holds in a strict sense. Such a $k$ is easy to find. If $\lfloor n \delta\rfloor=0$, use $k=n$, and for $\lfloor n \delta\rfloor>0$, use $k=0$.

Corollary. For weakly and strictly convex functions the theorem holds with its inequalities reversed.

To apply the theorem, all one has to know are the two parameters $n$ and $\delta$, i.e. compared to Jensen's inequality, there is no additional information needed. The only additional work comes from the evaluations of the floor, ceiling and sawtooth functions, which is manageable. Kaas and Buhrman (1980) show that any median $m$ of a binomial distribution with $n$ draws and probability of success $\delta$ falls within an interval with endpoints $\lfloor n \delta\rfloor$ and $\lceil n \delta\rceil$. Evaluating the floor and ceiling functions at $n \delta$ hence makes the successive eliminations in equations (3) and (4) obsolete.

\section{Application: Mating Market Clearing when Pater- nity is Uncertain}

Consider a society that is populated by single-period-lived fertile women and men. A woman who has mated at least once will give birth to exactly one child and her motherhood is certain. For her male partner(s), however, the probability of biological fatherhood $\delta$ is inversely related to the number of men the woman has mated: ${ }^{4}$

$$
\delta=\frac{1}{n_{\wp}}
$$

Let $\phi$ denote the sex ratio, i.e. the mass of the male population $\left(P_{\mathrm{o}^{\prime}}\right)$ divided by the mass of its female counterpart $\left(P_{\odot}\right)$. If we allow for real numbers of male partners per woman $\left(n_{\odot}\right)$ and female partners per man $\left(n_{\sigma^{*}}\right)$, the mating market would clear when $n_{q}$ multiplied by $P_{q}$ is equal to $n_{\sigma^{\prime}}$ times $P_{\sigma^{\prime}}$ :

$$
n_{q} P_{q}=n_{\sigma^{*}} P_{\sigma^{*}} \quad \Leftrightarrow \quad \frac{n_{q}}{n_{\sigma^{\prime}}}=\frac{P_{\sigma^{\prime}}}{P_{q}}(=\phi)
$$

\footnotetext{
${ }^{4}$ We only introduce those parts of the model necessary for the following welfare analysis. The interested reader is refered to Bethmann and Kvasnicka (2011) for a complete description of the model.
} 
Here, however, we rule out non-integer numbers for $n_{\varphi}$ and $n_{\sigma^{*}}$. Instead, we allow men to differ with respect to their number of female partners and introduce the average number of female partners per man $\bar{n}_{\sigma^{*}}$. Consider, for instance, the case when women mate exactly once. In that case, we will assume that a fraction $\{1 / \phi\}$ of men has access to $\lceil 1 / \phi\rceil$ female mating partners while the rest $1-\{1 / \phi\}$ has access to only $\lfloor 1 / \phi\rfloor$ partners. ${ }^{5}$ More generally, if women mate $n_{\varphi}$ times a fraction of $\left\{n_{\varphi} / \phi\right\}$ men has access to $\left\lceil n_{\varphi} / \phi\right\rceil$ female partners while a fraction of $1-\left\{n_{\odot} / \phi\right\}$ has only $\left\lfloor n_{\odot} / \phi\right\rfloor$ partners:

$$
\bar{n}_{\sigma^{*}}=\left\{\frac{n_{q}}{\phi}\right\}\left\lceil\frac{n_{q}}{\phi}\right\rceil+\left(1-\left\{\frac{n_{q}}{\phi}\right\}\right)\left\lfloor\frac{n_{q}}{\phi}\right\rfloor
$$

As a consequence, the mating market clearing condition reads as follows:

$$
\frac{n_{\odot}}{\bar{n}_{\sigma^{\prime}}}=\phi \quad \Leftrightarrow \quad \frac{n_{\odot}}{\phi}=\bar{n}_{\sigma^{\circ}}
$$

Assume that the sex ratio is equal to 0.8, that is there are four men for every five women in society. In case women mate only once, paternity is certain for every man. Moreover, one quarter of men would have two children while three quarters would have only one child. As a consequence, total male welfare $W(\phi, \delta)$ would be given by:

$$
W\left(\frac{4}{5}, 1\right)=\frac{3}{4} v(1)+\frac{1}{4} v(2)
$$

If, however, women mate twice, paternity becomes uncertain and men derive only expected utility. To be precise, one half of all men would mate twice and the other half thrice. In both cases, expected utility depends on a binomial distribution with probability of success $\delta=0.5$. With respect to the number of draws, however, both distributions differ: there are two for the former, and three for the latter. Let $\underline{\mathbb{E}}$ and $\overline{\mathbb{E}}$ denote the expectations operators under these two binomial distributions such that total male welfare is given by: ${ }^{6}$

$$
W\left(\frac{4}{5}, 2\right)=\frac{1}{2} \underline{\mathbb{E}}[v(k)]+\frac{1}{2} \overline{\mathbb{E}}[v(k)]
$$

To show that male welfare is higher when women mate only once, let us suppose the contrary were true, i.e. $W(0.8,1) \leq W(0.8,2)$. Applying $(7)$ from the theorem yields:

$$
\begin{aligned}
W\left(\frac{4}{5}, 1\right) & <\frac{1}{2} v(1)+\frac{1}{2}\left[\left\{\frac{3}{2}\right\} v\left(\left\lceil\frac{3}{2}\right\rceil\right)+\left(1-\left\{\frac{3}{2}\right\}\right) v\left(\left\lfloor\frac{3}{2}\right\rfloor\right)\right] \\
\Leftrightarrow \quad W\left(\frac{4}{5}, 1\right) & <\frac{3}{4} v(1)+\frac{1}{4} v(2)
\end{aligned}
$$

which contradicts (22). ${ }^{7}$ Note that using Jensen's inequality in (24) would not have led to a contradiction:

$$
W\left(\frac{4}{5}, 1\right)<\frac{1}{2} v(1)+\frac{1}{2} v\left(\frac{3}{2}\right) \quad \Leftrightarrow \quad \frac{1}{4} v(1)+\frac{1}{4} v(2)<\frac{1}{2} v\left(\frac{3}{2}\right)
$$

\footnotetext{
${ }^{5}$ We focus here on situations were differences among men are kept as small as possible.

${ }^{6}$ Because women are assumed to bear only one child, female welfare is always equal to $u(1)$.

${ }^{7}$ Note that the result can be generalized for all sex ratios $\phi$ and all $n_{\odot} \geq 2$ as follows: $W(\phi, 1) \geq W\left(\phi, n_{\odot}\right)$ with $n_{\text {}} \geq 2$.
} 
The last inequality is true for every strictly concave function and hence not helpful. Consider functions $f$ and $s$ in Figure 1. Inequality (7) suggests to use the point $s(n \delta)$ on the remaining linear segment as the upper bound for expected utility. This point is sufficient to lead to the contradiction. Jensen's inequality, in contrast, suggests to choose $f(n \delta)$ instead. However, $f(n \delta)$ exceeds $s(n \delta)$ whenever $n \delta \notin \mathbb{N}$ holds. As a consequence, it cannot lead to a contradiction.

\section{Conclusion}

In this note, I suggest an inequality for concave functions when the argument is drawn from a binomial distribution. The suggested inequality is tighter than Jensen's inequality and easily applicable. In particular, it does not require more information than Jensen's inequality. The usefulness of this new inequality is demonstrated in the context of mating market clearing

when paternity is uncertain. It is shown that it allows to derive a welfare result that would otherwise be impossible to show.

\section{References}

[1] Bethmann, D., Kvasnicka, M., 2011. The Institution of Marriage, Journal of Population Economics, 24(3), pp. 1005-1032.

[2] Kaas, R., Buhrman, J.M., 1980. Mean, Median and Mode in Binomial Distributions, Statistica Neerlandica 34(1), pp. 13-18. 